\title{
WSN Performance Issues and Various Clustering Methods
}

\author{
Anuja Modi \\ Computer Science and Engineering \\ VNS Faculty of Engineering \\ Bhopal, India
}

\author{
Gourishankar Prajapati \\ Head of Department Computer Science and \\ Engineering \\ VNS Faculty of Engineering \\ Bhopal, India
}

\begin{abstract}
There are several application have been developed with need of self organization for network. To fulfill this requirement need of wireless sensor network in such applications. To manage network efficiently clustering is used. Lots of works have been done in field of wireless sensor networks (WSNs) in last few years. These researches have boost potential of WSNs in applications such as security monitoring, disaster management, military area, border protection and health monitoring systems. Such applications are required to be remotely deployed sensor nodes in huge numbers and to operate autonomously. So there need to scalability, nodes are often collected into disjoint clusters. This paper, presents a categorization and common organization of available clustering proposal. This work analysis various clustering algorithms used for WSNs and give a review with focusing on their objectives features, etc. and proposed efficient clustering method for stable cluster formation and maintenance.
\end{abstract}

\section{Keywords}

WSNs Clustering, Cluster head selection, Clustering comparison.

\section{INTRODUCTION}

Wireless sensor network (WSN) has grown as one of attractive networking technologies now a day as its advantage of organization where lacking communication infrastructures. Sensor network is a network containing randomly placed sensor nodes and base stations (BS). The BS works as gateway to connect with public network or other network. WSNs offers efficient data collection, storage management, fast processing and access point to sensor nodes in its network. Sensor nodes are specially design to collect data for its environment and sends current data to BS. Still these nodes have limited power, processing and storage capacity. Hence routing processing and store route is difficult task due to limited resources and dynamically changing topology [1].

Research show their interest to minimizing and low-power design small sized battery applied sensors that capable to sense changing conditions as temperature, light and sound. Sensors normally design for data processing and communication with BS capability. Sensors have electronics circuit to convert environmental physical conditions into electrical signal. Every sensor node has radio communication system which is used to send and receive data to any node including BS. These characteristics attract practitioners to enhance limited capability for large network utilization to operate efficiently [2].

On disaster management circumstances for example earthquakes, sensor networks could helpful for particular map guided emergency services teams for properly functioning. Also military situations, health monitoring system and house protection system as shown in Fig. 1, sensor networks can be used. For any surveillance missions and to detect moving targets, chemical gases, or the presence of micro-agents sensor network and sensor nodes are effective technique. Sensor nodes are probable to be positioned randomly in any desired location uncontrolled manner, such as put down by any fly machine, and to together create a network in ad-hoc behavior [3]. Designing and operating such need large network is required scalable architectural and efficient management. Sensor nodes present in such type locations energy preserving is main constrain and their batteries not possible to recharged. For that reason, energy-aware algorithms design turn to be an essential issue for improving the life span of sensor nodes. Other application centric design objectives, e.g. high fidelity target detection and classification, are also considered.

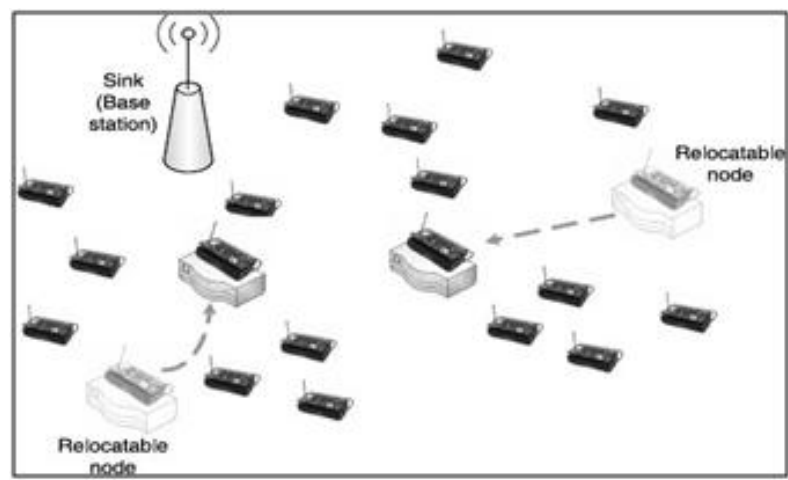

Fig. 1. An articulation of sample WSN architecture

Combination sensor nodes into clusters are taken by the research society to get the system scalability purpose. Each cluster should have a manager, known as the cluster-head $(\mathrm{CH})$. While several clustering algorithms have been given in the literature for ad-hoc networks [4], the point was primarily to create more stable clusters in environment with mobile nodes. Several techniques proposed try to make possible reach ability and route stability for network not worry about coverage. Recently clustering techniques proposed for WSNs [5]. These methods depending on node deployment schemes characteristics of $\mathrm{CH}$ node and network working model. A CH is selected by the sensor nodes in a cluster by the network members. CHs could create a second level network or just transmit the data to base-station

We ask that authors follow some simple guidelines. In essence, we ask you to make your paper look exactly like this document. The easiest way to do this is simply to download the template, and replace the content with your own material.

\section{PROBLEM IDENTIFICATION}

The design of routing protocols for WSNs is challenging because of several network constraints. WSNs suffer from the limitations of several network resources, for example, energy, 
bandwidth, central processing unit, and storage. The design challenges in sensor networks involve the following main aspects [6]-

- Limited energy capacity

- Sensor locations

- Limited hardware resources

- Massive and random node deployment

- Network characteristics and unreliable environment

- Data Aggregation

- Diverse sensing application requirements

- Scalability

WSNs do not use specialized routers for path discovery and traffic routing develops the wireless backbone architecture [7]. This means that certain nodes must be selected to communication. One of the general approaches to build up self-organize them is cluster based network architecture. This is achieved by partitioning ad hoc networks into clusters. Certain nodes, known as cluster-heads, would be responsible for the formation of cluster and maintenance of the topology of the network, and also for the resource allocation to all the nodes belonging to their clusters.

The mobile ad hoc network consists of nodes that move freely and communicate with each other. One way to support efficient communication between nodes is to partition ad hoc networks into clusters. Many clustering schemes have been proposed to form clusters. The WCA has improved performance compared with other previous clustering algorithms. However, the high mobility of nodes will lead to high frequency of re-affiliation which will increase the network overhead.

\section{WSNS CHARACTERISTICS AND OBJECTIVES}

The characteristics of WSNs and requirements have a important impact on the WSNs plan objectives for network capabilities and network performance [6].

\subsection{WSNs Characteristics}

As traditional wireless networks such as mobile ad hoc network (MANET) and cellular communication systems [8], WSNs have the following characteristics-

Density of Network: Sensor nodes are typically densely deployed and can be several orders of higher than a MANET.

Battery-power of sensor nodes: Sensor nodes are generally powered by battery and are positioned in an unusual location and it's difficult to modify or recharge their batteries power.

Limited energy, computation, and storage: Sensors nodes are having extremely limited resources as power energy, computation speed, and storage space abilities.

Self-configurable: Sensor nodes are deployed randomly and automatically organize themselves into a communication network.

Unreliability: Since sensor nodes have to deploy in extreme conditions so they may physical destroy or failures.

Data redundancy: Sensor nodes network almost deployed densely respective distance between nodes. Hence, the data sensed by nearest sensor nodes typically have a redundancy.
Specific Task: A sensor network is designed for a specific to perform special task. The installation requirements of a sensor network change with its application.

Many-to-one traffic pattern: Nearly all sensor networks, the data collected by nodes from multiple source sensor nodes sends to a particular base station, so many-to-one traffic pattern follows in WSNs.

Dynamic topology: Network topology transforms regularly if any node get fails, damage, add, energy lost, or channel broken down.

\subsection{WSNs Objectives}

Following major WSNs plan objectives [9]-

Small node size: small node size nodes are easy to node deployment in any type of environment. If size is small it also reduces the power spend and cost of sensor nodes.

Low node cost: In many cases sensor nodes remain no more reusable, so reducing cost of sensor nodes is very essential and thus whole network cost could be reduced.

Low power consumption: As known sensor nodes are used by their battery power and it is very difficult or impossible to recharge their batteries, it's important to save the power consumed by sensor nodes so life span of nodes, as well as the whole network can be extended.

Scalability: The number sensor nodes in WSNs need of tens, hundreds, or thousands, according to application of network requirements, so sensor networks should be scalable for different network sizes.

Reliability: WSNs must provide error control and correction to ensure consistent data delivery in presence of noisy, error etc.

Self-configurability: WSNs once install, sensor nodes should be able to in parallel organize themselves to form network and manage them self event of topology changes of node crashed.

Adaptability: In sensor networks, a node may crash, join, or move away, which would result dynamic node density and topology. Hence WSN should be adaptive in nature to handle such changes or problem.

Channel utilization: WSNs have limited bandwidth or resources so WSNs should capably to use bandwidth in such manner to proper channel utilization.

Fault tolerance: Sensor nodes may fails as crucial conditions environments. Thus, sensor nodes should be manage fault and must have ability of self testing, self-repairing and selfrecovering.

Security: A sensor network should have security techniques to prevent the data information in the network or a sensor node from unauthorized access or attacks.

\section{RELATED WORK}

In flat networks, each node typically plays the same role and sensor nodes collaborate together to perform the sensing task. Due to the large number of such nodes, it is not feasible to assign a global identifier to each node. This consideration has led to data centric routing [10], where the BS sends queries to certain regions and waits for data from the sensors located in the selected regions.

Hierarchical or cluster-based routing, are well-known techniques with special advantages related to scalability and efficient communication. As such, the concept of hierarchical 
routing is also utilized to perform energy-efficient routing in WSNs. During application of this type architecture more energy nodes may utilized for processing and send sensed information transmission. Many protocols have been given for such methodology like LEACH [11], PEGASIS [12], TEEN [13] and APTEEN [14].

Load is important factor for clustering technique by keep in mind author in paper [15] mention load balanced clustering method for wireless network. This algorithm based on distance and density of nodes within a network, this feature makes this method different from given in previously. It is a balanced cluster and utilizes power of all nodes properly for WSNs of non-uniform distribution, taking into account optimal configuration of clusters. This method algorithm can form more stable and reasonable cluster structure, and also improve the network life cycle significantly.

WCA [16] is a classical algorithm based on node degree, the number of single-hop neighbors. The election of cluster head relies upon the factors of node degree, send-receive energy and residual energy. Meanwhile the size of cluster (the communication consumes large amounts of energy when cluster is too large) is limited in order to save energy. In contrast, the WCA clustering algorithm is more comprehensive than the previously proposed algorithms, and some experiments show that the performance is more superior. Additionally, WCA need to calculate weight of every node so all nodes have to keep record of all information regarding decision parameters before start network as well as clustering phase. Hence extra effort have to apply before communication and resources are utilized for controlling. In the process of aggregating and forwarding, the overhead may also give rise to excessive energy consumption and rapid death of cluster head node, incurring instability in the network topology.

K-clustering [17] algorithm can comprise highest k-hop nonoverlapping clusters with fractional networks topology information somewhat than the complete network topology. At the similar time, it can also save energy to prolong network survival time. Furthermore, owing to dynamic network topology changes, it is of significance studying clustering based on local information. Nevertheless, it doesn't consider cluster size and may form unbalanced cluster. For example, some clusters contain tremendous number of nodes, which results in too large overhead of inter communication.

ESAC [18] algorithm combines the advantages of the above proposed algorithms, and it improves clustering performance by overcoming their shortcomings. This algorithm uses the method of calculating weight in selecting cluster head. The weight of each node is calculated relying on the combination of two parameters: residual energy and mobility. The cluster size ranges between two and the distance between each cluster node and its cluster head is no more than 2-hop. This differs from $\mathrm{LEACH}$, and the algorithm builds the balanceable and smooth clustering network by considering the k-density, residual energy and mobility so as to avoid fixed cluster head project, which may results in excessive energy consumption of cluster head. The process of electing cluster head is relaunched in a certain period (service period). It calculates the weight of each node in every stage of cluster head building in order to ensure the most appropriate node to become cluster head and restrict the size of cluster very well. When a cluster head dies or is moved to other cluster, the maintenance process is triggered. The process is similar to that of building cluster launched by a random member of the former cluster, and is limited only to the members losing their cluster head. In this way it can avoid the previous 'chain loop' problem existed in clustering algorithm, and has little effect on the network topology. However, the structure of 2-hop clusters is not suitable for all circumstances. In some cases, we need to constitute clusters more than 2-hop.

\section{CLUSTERING ALGORITHMS FOR WSNS}

\subsection{Linked Cluster Algorithm (LCA)}

The works have given is among the first ones on clustering of wireless networks. The main focus is principally on forming associate effective topology which will handle the roaming of nodes. By clustering, $\mathrm{CHs}$ are hoped to create a backbone network to that cluster members will connect whereas on the move. The target of the planned distributed method is to create clusters specified a $\mathrm{CH}$ is directly connected to all or any nodes in its cluster. LCA is therefore geared for increasing network connection. The method assumes synchronal nodes and time-based medium access. A node is allotted the find time for the frame that matches its ID. First, every node broadcasts its ID and listens to transmission of alternative nodes. Within the next phase, a node broadcast the set of neighbors that it received from and therefore each node can eventually recognize its 1-hop and 2-hop neighbors. A node $\mathrm{x}$ becomes a $\mathrm{CH}$ if it's maximum ID among its neighbors or doesn't have maximum ID in its 1-hop neighborhood, however there exists a minimum of one neighboring node y specified $\mathrm{x}$ is that the highest ID node in y's 1-hop neighborhood. Since LCA is found to yield excessive quantity of clusters. The thought is to choose a node $\mathrm{x}$ randomly because the initial $\mathrm{CH}$ and assign its neighbors to such initial cluster. Then the node y with minimum ID within the cluster is nominative as a $\mathrm{CH}$. The neighbors of $\mathrm{y}$ that don't seem to be approachable to $\mathrm{x}$ would be a part of the second cluster. The procedure is continual for the third cluster so on.

\subsection{CLUBS}

In paper [19], projected CLUBS, associate degree rule that forms clusters through native broadcast and converge in an exceedingly time proportional to the native density of nodes. Basically, cluster formation in CLUBS relies on the subsequent 3 characteristics:

- Each node within the network should be connected to a cluster.

- Most diameters of all clusters within the network ought to be same.

- Clusters ought to support the intra-cluster communication, which suggests nodes in an exceedingly cluster should be able to communicate with every others.

\subsection{Ranked Management Clustering}

Unlike most of the revealed schemes, the goal of Banerjee and Khuller is to create a multi-tier ranked clustering [20]. Variety of cluster's properties like cluster size and therefore the degree of overlap that square measure helpful for the management and quantify of the hierarchy, are thought-about whereas grouping the nodes. Within the projected theme, any node within the WSN will initiate the cluster formation method. Leader with least node ID can take precedence, if multiple nodes started cluster formation method at an equivalent time. The rule yield in 2 phases: Tree discovery and Cluster formation. 


\subsection{Random Competition Primarily based Clustering (RCC)}

Although RCC [21] is meant for mobile accidental networks, it's conjointly applicable to WSNs. RCC chiefly focuses at cluster stability so as to support mobile nodes. The RCC rule applies the primary Declaration Wins rule, within which any node will "govern" the remainder of the nodes in its radio coverage if it's the primary to assert being a $\mathrm{CH}$. when hearing the claim that is broadcasted by the primary node, neighboring nodes be a part of its cluster as member and provides up their right to be a $\mathrm{CH}$. to keep up clusters, each $\mathrm{CH}$ within the network broadcast a $\mathrm{CH}$ claim packet sporadically. Since there's a time delay between broadcasting a claim packet and receiving it, synchronal broadcast will probably produce a conflict. Being unaware of on-going claims, several neighboring nodes might broadcast $\mathrm{CH}$ claim packets at the same time. To avoid such a retardant RCC expressly employs a random timer and uses the node ID for arbitration. every node within the network reset its random continuance, each time before broadcasting its $\mathrm{CH}$ claim packet. throughout this random time if it receives a broadcast message carrying $\mathrm{CH}$ claim packet from another node, it merely ceases the transmission of its $\mathrm{CH}$ claim. Since random timer isn't a whole resolution, RCC resolve any the synchronal broadcast issues by victimization the node ID. If the conflict persists, node having lower ID can become the $\mathrm{CH}$. though frequent node quality still has direct impact, RCC is shown to be additional stable than typical clustering schemes like [21]. A $\mathrm{CH}$ in adaptative clustering abandons its role once it hears a node with a lower ID, while, a CH in RCC solely offers up its position once another $\mathrm{CH}$ moves just about it

\subsection{GS3}

Work in [22], gift associate degree rule, called GS3, for selfconfiguring a wireless network into a cellular polygon structure. The authors argue that ignoring the geographical boundary of clusters will be unwise; particularly for terribly giant network. They outline the radius of the circle that contains all nodes within the cluster as a live for the geometric size. An outsized cluster radius is alleged to extend energy consumption and responsibility for intra-cell communication and limit the special reprocess of radio frequencies within the network. Two types of nodes square measure assumed within the system: huge and little. The massive nodes square measure chargeable for initiating the cluster formation method. Additionally, they interface the little nodes to alternative cells and alternative network, e.g. the net and act as negotiator. It ought to be noted that the cellular polygon structure is virtual and is simply wont to guide the grouping and distribution of nodes. to create the cellular polygon structure, the world is split into cells of equal radius R. one in all the massive nodes starts the clustering method by choosing the heads of neighboring cells that choose their neighbors so on. random members become cell members. Upon their choice cell heads relocate to the centers of their cells and begin establishing their neighboring cells by choosing their heads. theThe method is recurrent till no additional cells can be more.

\subsection{Energy Economical Ranked Clustering (EEHC)}

Paper [23] projected EEHC; a distributed, irregular clustering rule for WSNs with the target of increasing the network life. $\mathrm{CHs}$ collected the sensors' readings in their individual clusters associate degreed send and mass report back to the base- station. Their technique relies on 2 stages; initial and extended. Within the initial stage, conjointly referred to as single-level clump, every device node announces itself as a $\mathrm{CH}$ with chance $\mathrm{p}$ to the neighboring nodes at intervals its communication varies. These $\mathrm{CHs}$ square measure named because the volunteer $\mathrm{CHs}$. All nods that square measure at intervals $\mathrm{k}$ hops vary of a $\mathrm{CH}$ receive this announcement either by direct communication or by forwarding. Any node that receives such announcements and isn't itself a $\mathrm{CH}$ becomes the member of the current cluster. Forced $\mathrm{CHs}$ square measure nodes that square measure neither $\mathrm{CH}$ nor belong to a cluster. If the announcement doesn't reach to a node at intervals a planned measure $t$ that's calculated supported the length for a packet to succeed in a node that's $\mathrm{k}$ hops away, the node can become a forced $\mathrm{CH}$ forward that it's not at intervals $\mathrm{k}$ hops of all volunteer $\mathrm{CHs}$.

\subsection{Low Energy Adaptative Clustering Hierarchy (LEACH)}

LEACH is one in all the foremost fashionable clustering algorithms for WSNs [20]. It forms clusters supported the received signal strength and uses the $\mathrm{CH}$ nodes as routers to the base-station. All information process such as data fusion and aggregation are native to the cluster. LEACH forms clusters by employing a distributed rule, wherever nodes build autonomous choices with none centralized management. An initial a node decides to be a $\mathrm{CH}$ with a chance $\mathrm{p}$ and broadcasts its call. Every non- $\mathrm{CH}$ node determines its cluster by selecting the $\mathrm{CH}$ which will be reached victimization the smallest amount communication energy. The role of being a $\mathrm{CH}$ is revolved sporadically among the nodes of the cluster so as to balance the load. The rotation is performed by obtaining every node to settle on a random range " $\mathrm{T}$ " between zero and one.

Fig. 2. State transition for the FLOC clustering algorithm.

\subsection{Quick Native Clustering Service (FLOC)}

FLOC [24] could be a distributed technique that produces about equal sized clusters with minimum over-lap. The assumed radio model classifies nodes supported their proximity to the $\mathrm{CH}$ into inner (i-band) and outer (o-band). Iband nodes can suffer little interference act with the $\mathrm{CH}$, whereas message from o-band nodes could also be lost. Material favors i-band membership so as to extend the hardiness of the intra-cluster traffic. A node stays idle looking ahead to some random length to receive letter of invitation from any potential $\mathrm{CH}$. If the node gets no invite, it becomes a candidate $\mathrm{CH}$ and broadcasts a movement message (transition 1). Upon hearing the movement message a recipient node " $k$ " that's already associate degree i-band member of a cluster $\mathrm{Ck}$, can reply back to tell the candidate $\mathrm{CH}$ regarding such membership. Fig. a pair of summarizes the material rule. The candidate $\mathrm{CH}$ can then understand the conflict associate degreed be a part of $\mathrm{Ck}$ as an o-band node (transition 3). If the candidate $\mathrm{CH}$ receives no conflict messages, it becomes a $\mathrm{CH}$ and starts invitatory members to its cluster (transition 4). Associate degree idle node would be a part of a cluster as associate degree o-band node (transition 5) if it doesn't receive letter of invitation from a better $\mathrm{CH}$ (transition 2). That call will be modified, if the node later receives letter of invitation from a better $\mathrm{CH}$, i.e. the node switch its membership to a far better cluster rule for Cluster. 
Table 1. Comparison of the existing Clustering Algorithms

\begin{tabular}{|c|c|c|c|c|c|c|c|c|}
\hline $\begin{array}{c}\text { Clustering } \\
\text { approaches }\end{array}$ & $\begin{array}{c}\text { Convergence } \\
\text { time }\end{array}$ & $\begin{array}{c}\text { Node } \\
\text { mobility }\end{array}$ & $\begin{array}{c}\text { Cluster } \\
\text { Overlapping }\end{array}$ & $\begin{array}{c}\text { Location } \\
\text { awareness }\end{array}$ & $\begin{array}{l}\text { Failure } \\
\text { recovery }\end{array}$ & $\begin{array}{l}\text { Balanced } \\
\text { clustering }\end{array}$ & $\begin{array}{l}\text { Cluster } \\
\text { stability }\end{array}$ & $\begin{array}{l}\text { Power } \\
\text { Saving }\end{array}$ \\
\hline LCA & Variable $\mathrm{O}(\mathrm{n})$ & Possible & No & Required & Yes & OK & Moderate & No \\
\hline Adaptive clustering & Variable $\mathrm{O}(\mathrm{n})$ & Yes & No & Required & Yes & OK & Low & N/A \\
\hline CLUBS & Variable $\mathrm{O}(\mathrm{n})$ & Possible & High & $\begin{array}{c}\text { Not } \\
\text { required }\end{array}$ & Yes & OK & Moderate & N/A \\
\hline $\begin{array}{l}\text { Hierarchical control } \\
\text { clustering }\end{array}$ & Variable $\mathrm{O}(\mathrm{n})$ & Possible & Low & $\begin{array}{c}\text { Not } \\
\text { required }\end{array}$ & Yes & Good & Moderate & N/A \\
\hline $\mathrm{RCC}$ & Variable $\mathrm{O}(\mathrm{n})$ & Yes & No & Required & Yes & Good & Moderate & N/A \\
\hline GS3 & Variable $\mathrm{O}(\mathrm{n})$ & Possible & Low & Required & Yes & Good & Moderate & N/A \\
\hline EEHC & $\begin{array}{c}\text { Variable } \\
\mathrm{O}(\mathrm{k} 1+\mathrm{k} 2+. .+\mathrm{kh})\end{array}$ & No & No & Required & N/A & OK & N/A & Yes \\
\hline LEACH & Constant $\mathrm{O}(1)$ & Fixed BS & No & $\begin{array}{c}\text { Not } \\
\text { required }\end{array}$ & Yes & OK & Moderate & No \\
\hline FLOC & Constant $\mathrm{O}(1)$ & Possible & No & $\begin{array}{c}\text { Not } \\
\text { required }\end{array}$ & Yes & Good & High & N/A \\
\hline HEED & Constant $\mathrm{O}(1)$ & Stationary & No & $\begin{array}{c}\text { Not } \\
\text { required }\end{array}$ & N/A & Good & High & Yes \\
\hline DWEHC & Constant $\mathrm{O}(1)$ & Stationary & No & Required & N/A & $\begin{array}{l}\text { Very } \\
\text { good }\end{array}$ & High & Yes \\
\hline MOCA & Constant $\mathrm{O}(1)$ & Stationary & Yes & $\begin{array}{c}\text { Not } \\
\text { required }\end{array}$ & N/A & Good & High & Yes \\
\hline $\begin{array}{l}\text { Attribute-based } \\
\text { clustering }\end{array}$ & Constant $\mathrm{O}(1)$ & No & No & $\begin{array}{c}\text { No } \\
\text { Required }\end{array}$ & Yes & $\begin{array}{l}\text { Very } \\
\text { good }\end{array}$ & High & Yes \\
\hline
\end{tabular}

\subsection{Hybrid Energy-Efficient Distributed Clustering (HEED)}

HEED [25] is a distributed clustering scheme in which $\mathrm{CH}$ nodes are picked from the deployed sensors. HEED considers a hybrid of energy and communication cost when selecting CHs [26]. Unlike LEACH, it does not select cell-head nodes randomly. Only sensors that have a high residual energy can become cell-head nodes. HEED has three main characteristics:

- The probability that two nodes within each other's transmission range becoming $\mathrm{CHs}$ is small. Unlike

- $\mathrm{LEACH}$, this means that $\mathrm{CHs}$ are well distributed in the network.

- Energy consumption is not assumed to be uniform for all the nodes.

- For a given sensor's transmission range, the probability of $\mathrm{CH}$ selection can be adjusted to ensure inter- $\mathrm{CH}$ connectivity.

\subsection{Distributed Weight-Based Energy- Efficient Hierarchical Clustering (DWEHC)}

Paper [27] mentions DWEHC method to overcome drawbacks of HEED algorithm. This method creates an equilibrium cluster size for best intra clustering formation. DEHC function in distributed environments with constant time complexity. This method performs clustering within constant time complexity. Every node checks its direct connected node by using this information it derives its weight for current state.

This weight is an important parameter for wireless nodes to save neighbor nodes energy. Among these adjacent nodes highest weight able to select as $\mathrm{CH}$ and all other nodes becomes members of cluster. At this situation nodes are known as 1-level nodes if any nodes have direct reach ability to $\mathrm{CH}$. Every node optimizes this function to reach $\mathrm{CH}$ with lowest energy used. Any node communicates with its neighbor node to calculate minimum cost route to access $\mathrm{CH}$ node. Neighbor nodes send its distance to $\mathrm{CH}$ and if node notable for direct communication then its second level node. This also called two hop distance path from $\mathrm{CH}$. Nodes can change its cluster $\mathrm{CH}$ if other $\mathrm{CH}$ reaches ability with one hop. This process repeated till best energy saving topology formed for intra clustering process. But if level of $\mathrm{CH}$ accessibility is high than energy consumption will increase for communication so need to restricts levels, for this purposed max size of cluster is defined not cluster can make large cluster as max cluster size.

\subsection{MOCA}

Generally all previous algorithm works only on to reducing cluster numbers within a wireless network. The work given in paper [28] sure that few number of overlap clusters use full to service lots of facilities such as connectivity to different clusters, topology management also recovery of cluster head failure. This work demonstrate MOCA algorithm, based on random with distributed cluster including multi-hop and overlapping clusters. The primary objective of this technique 
is to guarantee that every wireless node is $\mathrm{CH}$ or $\mathrm{K}$-hop distance from cluster head where $\mathrm{K}$ is predefined fixed number. This $\mathrm{K}$ define radius of cluster.

\subsection{Load-Balanced Clustering Algorithm with Distributed Self-Organization}

Load balancing in distributed environment is given in paper [15]. This method helps for self configuration of WSNs if any network is not uniform in nature. It maintains optimality of cluster size with its all working features. This method is very effective on compare to other existing techniques given. This method is capable to create more stable and minimum cost expense which helps to improve overall lifetime of wireless network

\section{CONCLUSION}

In last few decades wireless network has attracts many user as its unique features. A lots of applications are working on such type of network not only like civilian but also military application are depends on WSNs. There are many situations where other wired or radio networks are not able to perform communication very well such as unusual and remote areas including rural fields. Whenever natural disaster like floods or earthquake occur this type of network works very well. Border security, battle field monitoring in such situations WSNs can perform task efficiently as compare to other networks. Any wireless network may consist of lots of sensor to sense the information. All these nodes need to manage them carefully and configure them self automatically. To fulfill such duties a good clustering technique is very essential this increases stability of WSNs and life cycle of existing network. Hence lot of work done in this field in last two decades.

This paper, review presented research work and gives contrast of recent schemes. This gives categorization respective attributes required for efficient WSNs design. In this paper we have presented an enhanced weight based clustering algorithm to improve upon their stability and to reduce re-affiliation of the nodes. This work focuses on reducing the instability caused by high-speed moving nodes, by taking relative mobility of node and its neighbors into consideration. Since WCA support stable cluster head election and the disadvantage is re-affiliation of nodes which is reduced by mobility prediction, it results in stable clustering.

This work may extend to design better clustering method which can perform efficiently in wireless environment. This comparative study helps to find novel method for node power utilization minimization and improve life time of overall network. In future clustering method will be identifying for power management and reliable topology.

\section{REFERENCES}

[1] K. Akkaya, M. Younis, A survey of routing protocols in wireless sensor networks, Elsevier Ad Hoc Network Journal 3/3 (2005) 325-349.

[2] C-Y. Chong, S.P. Kumar, Sensor networks: evolution, opportunities, and challenges, Proceedings of the IEEE 91 (8) (2003) 1247- 1256.

[3] R. Min, et al., Low power wireless sensor networks, in: Proceedings of International Conference on VLSI Design, Bangalore, India, January 2001.

[4] V. Kawadia, P.R. Kumar, Power control and clustering in Ad Hoc networks, in: Proceedings of IEEE INFOCOM, San Francisco, CA, March 2003.
[5] O. Younis, S. Fahmy, HEED: A Hybrid, EnergyEfficient, Distributed clustering approach for Ad Hoc sensor networks, IEEE Transactions on Mobile Computing 3 (4) (2004) 366-379.

[6] Jun Zheng and Abbas Jamalipour, "Wireless Sensor Networks: A Networking Perspective", a book published by A John \& Sons, Inc, and IEEEE, 2009.

[7] C. Karthik Sendhil Kumar, R.Sukumar, M.Nageswari, "Sensors Lifetime Enhancement Techniques in Wireless Sensor Networks - A Critical Review", IRACST International Journal of Computer Science and Information Technology \& Security (IJCSITS), ISSN: 2249-9555 Vol. 3, No.2, April 2013.

[8] Gajendra Singh Deepa Upadhyay, Kapil Vyas, "Clustering Algorithm in Manets for Stable Cluster Recent Trends Survey", International Journal of Advanced Research in Computer Science and Software Engineering Volume 4, Issue 9, September 2014.

[9] Shio Kumar Singh, M P Singh and D K Singh, "Routing Protocols in Wireless Sensor Networks - A Survey", International Journal of Computer Science \& Engineering Survey (IJCSES) Vol.1, No.2, November 2010.

[10] I.F. Akyildiz et al., Wireless sensor networks: a survey, Computer Networks 38 (2002) 393-422.

[11] W. Heinzelman, A. Chandrakasan,H. Balakrishnan, Energy-efficient communication protocol for wireless microsensor networks, in: Proceedings of the 33rd Hawaii International Conference on System Sciences (HICSS'00), January 2000.

[12] S. Lindsey, C.S. Raghavendra, PEGASIS: power efficient gathering in sensor information systems, in: Proceedings of the IEEE Aerospace Conference, Big Sky, Montana, March 2002.

[13] Manjeshwar, D.P. Agrawal, TEEN: a protocol for enhanced efficiency in wireless sensor networks, in: Proceedings of the 1st International Workshop on Parallel and Distributed Computing Issues in Wireless Networks and Mobile Computing, San Francisco, CA, April 2001.

[14] Manjeshwar, D.P. Agarwal, APTEEN: a hybrid protocol for efficient routing and comprehensive information retrieval in wireless sensor networks, in: Parallel and Distributed Processing Symposium. Proceedings International, IPDPS 2002, pp. 195-202.

[15] Ying Liao, Huan Qi, and Weiqun Li, "Load-Balanced Clustering Algorithm with Distributed Self-Organization for Wireless Sensor Networks", IEEE Sensors Journal, Vol. 13, No. 5, May 2013.

[16] M. Chatterjee, S. K. Das, and D. Turgut, "WCA: A weighted clustering algorithms for mobile ad hoc networks,"Cluster Comput., vol. 5, no. 2, pp. 193-204, 2002.

[17] Y. Fernandess and D. Malkhi, "K-clustering in wireless ad-hoc networks," in Proc. 2nd ACM Workshop Principles Mobile Comput. Conf., Oct. 2002, pp. 31-37.

[18] M. Lehsaini, H. Guyennet, and M. Feham, "A novel cluster-based selforganization algorithm for wireless 
sensor networks," inProc. Int. Symp. Collabor. Technol. Syst. Conf., May 2008, pp. 19-26

[19] R. Nagpal, D. Coore, An algorithm for group formation in an amorphous computer, in: Proceedings of the 10th International Conference on Parallel and Distributed Systems (PDCS'98), Las Vegas, NV, October 1998.

[20] W.B. Heinzelman, A.P. Chandrakasan, H. Balakrishnan, Application specific protocol architecture for wireless microsensor networks, IEEE Transactions on Wireless Networking (2002).

[21] K. Xu, M. Gerla, A heterogeneous routing protocol based on a new stable clustering scheme, in: Proceeding of IEEE Military Communications Conference (MILCOM 2002), Anaheim, CA, October 2002.

[22] H. Zhang, A. Arora, GS3: scalable self-configuration and self-healing in wireless networks', in: Proceedings of the 21st ACM Symposium on Principles of Distributed Computing (PODC 2002), Monterey, CA, July 2002.

[23] S. Bandyopadhyay, E. Coyle, An energy efficient hierarchical clustering algorithm for wireless sensor networks, in: Proceedings of the 22nd Annual Joint Conference of the IEEE Computer and Communications Societies (INFOCOM 2003), San Francisco, California, April 2003.
[24] M. Demirbas, A. Arora, V. Mittal, FLOC: a fast local clustering service for wireless sensor networks, in: Proceedings of Workshop on Dependability Issues in Wireless Ad Hoc Networks and Sensor Networks (DIWANS'04), Palazzo dei Congressi, Florence, Italy, June 2004.

[25] O. Younis, S. Fahmy, HEED: A Hybrid, EnergyEfficient, Distributed clustering approach for Ad Hoc sensor networks, IEEE Transactions on Mobile Computing 3 (4) (2004) 366-379.

[26] Jun Wang, Xuegang Zhu,Yong Cheng and Yongsheng Zhu, "A Distributed, Hybrid Energy-Efficient Clustering Protocol for Heterogeneous Wireless Sensor Network" International Journal of Grid and Distributed Computing Vol. 6, No. 4, August, 2013.

[27] P. Ding, J. Holliday, A. Celik, Distributed energy efficient hierarchical clustering for wireless sensor networks, in: Proceedings of the IEEE International Conference on Distributed Computing in Sensor Systems (DCOSS'05), Marina Del Rey, CA, June 2005.

[28] Youssef, M. Younis, M. Youssef, A. Agrawala, Distributed formation of overlapping multi-hop clusters in wireless sensor networks, in: Proceedings of the 49th Annual IEEE Global Communication Conference (Globecom'06), San Francisco, CA, November 2006. 